\title{
Cancer History Present
}

National Cancer Institute

\section{Source}

National Cancer Institute. Cancer History Present. NCI Thesaurus. Code C160182.

An indication that a cancer history is present. 